\title{
A Brief Analysis of the Dual Influences of Speech Recognition Assistance on Simultaneous Interpretation
}

\author{
Xiaolin Wang ${ }^{1}$

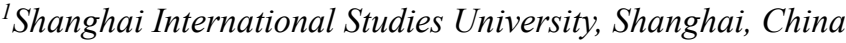 \\ 926069396@qq.com

\begin{abstract}
In the era of artificial intelligence, big data, speech recognition, machine translation and other technologies have brought an increasing impact on the field of manual translation. On the one hand, it challenges manual translation, and on the other hand, it assists and improves manual translation. The application of speech recognition software in simultaneous interpretation is a hot topic. Based on the study and review of previous research results, this article analyzes the positive and negative effects of speech recognition technology on the interpretation process in simultaneous interpretation based on the author's experience, and provide some reference value for the use of speech recognition assistance in simultaneous interpretation for the author and other interpreters.
\end{abstract}

Keywords: Simultaneous Interpretation, Speech Recognition Technology, Interpretation

\section{浅析语音识别辅助对同声传译翻译过程的双重影响}

\author{
王小琳 $^{1}$
}

\author{
${ }^{1}$ 上海外国语大学俄语口译专业, 上海, 中国 \\ 926069396@qq.com
}

摘要: 人工智能时代, 大数据、语音识别、机器翻译等技术给人工翻译领域带来越来越大的影响, 一方面冲击 着人工翻译, 另一方面辅助和完善了人工翻译。语音识别软件在同声传译中的应用是一个热点议题, 本文在学 习和查阅前人研究结果的基础上，结合自身经验，分析同声传译中语音识别技术对翻译过程的积极和消极影 响，为笔者以及其他译员对于在同声传译中使用语音识别辅助提供一些参考价值。

关键词: 同声传译; 语音识别技术; 翻译

1. 引言

随着经济社会全球化的发展, 社会语言服务需求 也在不断增长。人工智能翻译技术的应用在语言服务 业中发挥着越来越重要的作用。在越来越多的会议中 出现机器翻译、语音识别技术和同步多语字幕翻译技 术。而语音识别技术的辅助, 改变了译员传统的工作 模式, 有助于改善现场翻译质量, 减轻译员心理压力。 同时, 对语音识别技术的应用还不广泛的情况下, 译 员与语音识别技术之间的配合还存在一些问题, 这些 问题会对翻译质量造成负面影响, 因此, 探究语音识 别技术辅助的合理运用具有重要意义。

\section{2. 同声传译的基本概念及基本特点}

\section{1. 同声传译的基本概念}

同声传译，简称 “同传”，又称 “同声翻译”、“同 步口译”, 是指译员在不打断讲话者讲话的情况下, 不间断地将内容口译给听众的一种翻译方式, 同声传 译员通过专用的设备提供即时的翻译, 这种方式适用 于大型的研讨会和国际会议, 通常由两名到三名译员 轮换进行。

\section{2. 同声传译的基本特点}

同声传译学术性较强, 对口译员素质要求较高。 
由于同声传译广泛应用于各种大型会议和研讨会, 发 言内容较为正式和专业, 因此口译员在翻译的时候, 应当保持译文的流畅性和专业性。

同声传译的翻译滞后于听辨，因此需要适当分配 精力。一般情况下，译员翻译的时间落后于听辨时间 2-10 秒或者是三个单词, 也就是说, 在翻译前一句内 容时, 又要同时听辨下一句内容, 还需要适当联想发 言人的发言内容, 同时, 对信息准确度要求高的内容 需要记录, 例如数字、人名、职称等等。同一时间要 完成多项任务, 这就要求口译员要适当分配精力, 以 听辨为主。

同声传译具有即时性, 这就造成了同声传译的不 可修正性, 即译文输出后, 重新勘酷加以改正的可能 性很小, 口译员精力大部分都分配在后面内容的听辨 中, 一味纠结前一句输出译文的质量对后面的译文会 带来负面影响，导致信息遗漏等问题。

\section{3. 语音识别技术在同声传译中的应用}

\section{1. 语音识别技术的发展}

语音识别技术就是把语音信号转变为相应的命 令, 从而使计算机能够理解的一项技术。语音识别技 术的研究最早开始于 20 世纪 50 年代, 20 世纪 90 年 代以来第一次掀起研究和产业应用的高潮。(王海坤, 潘嘉, 刘聪, 2018) 进入 90 年代后, 语音识别技术 开始应用于全球市场, 许多著名科技互联网公司, 如 IBM, Apple 等, 都为语音识别技术的开发和研究投 入巨资。到了 21 世纪, 语音识别技术研究重点转变 为即兴口语与自然对话以及多语种的同声翻译。

\section{2. 语音识别技术在同声传译中的应用}

语音识别技术虽然在长期的发展过程中取得了 有了历史性的进展, 但是仍存在一些技术上的不足。 语音识别过程中的噪声处理仍然是一个很难突破的 难题, 并且超过一定的距离机器就很难识别使用者的 语言, 甚至是停止工作不进行识别工作, 不仅有距离 上的影响, 当面临于嘈杂的环境中, 系统也很难辨析 出讲话人的语言。同时, 讲话人的口音和发言不准确 也会对语音识别转化的文本产生影响。现阶段语音识 别技术在同声传译中的应用仍然不广泛。(于晓明, 2019)

笔者曾有过一次在同声传译中应用语音识别技 术辅助的亲身体验。在参加一次语音识别辅助队译员 翻译过程影响的实验中, 笔者面前放置一台电脑, 电 脑屏幕上会自动出现经语言识别系统转化过的源语 文本, 笔者可以选择看屏幕进行翻译或者根据自己笔 记进行翻译。在整个翻译过程中, 笔者无法兼顾两种 提示方式, 只能专注于自己笔记或者只专注于电脑屏 幕的提示。但是在以电脑屏幕为主要辅助工具时, 笔 者的翻译方式由听译转变为视译, 难以处理好听和看
的关系, 精力分配出现问题。所以整个翻译过程中, 笔者更多依赖电脑屏幕上的源语文本。事后在总结经 验时, 笔者得出几点结论: 对于翻译水平有限的译员, 语音识别系统的辅助起到很大的积极作用, 此时以视 译为主, 听译为辅。在初次使用语音识别辅助的过程 中, 要事先定位好自己的主要翻译辅助工具, 即专注 于电脑屏幕还是笔记, 否则会出现混乱。以笔记为主 要辅助工具时, 如果在听辨过程中有信息遗漏, 在电 脑屏幕上寻找遗漏信息的记录时, 会导致注意力被吸 引, 耗时过长, 影响对下文的听辨和翻译。

\section{4. 语音识别辅助对同声传译翻译过程的双重 影响}

\section{1. 语音识别辅助对同声传译翻译过程的积 极影响}

\section{1. 1. 提高译文完整性}

同声传译对译员注意力要求很高, 精力分配不当 时很容易导致输出译文质量的低下。听辨精力分配不 足, 则导致信息遗漏, 这时便可以利用语音识别系统 的辅助作用, 迅速在电脑屏幕源语转换文本中搜索遗 漏信息。通过语音识别系统转换的源语文本信息全面, 可以让译员更轻易的把握逻辑关系和关键信息。在只 靠听辨和笔记的过程中, 来不及翻译的源语经常被忘 记和遗漏, 而借助涵盖大部分甚至全部内容的源语转 换文本，可以提高译文的完整性，且允许在时间足够 的情况下对之前遗漏的信息不着痕迹地适当补充, 提 高翻译质量。

\subsection{2. 减轻听辨压力}

同声传译中译员需要一心多用, 相比较于交替传 译和视译, 同声传译接受的语流更为碎片化和随机化, 讲者通常会在无意识的情况下反复重复前文内容。

（李天韵, 2018）语音识别系统如能提供精确度较高 的源语转换文本, 译员可以将语音识别系统作为主要 的辅助工具, 由原来的听译为主, 笔记视译为辅, 转 换为视译为主, 听译为辅, 用听译来辨别和校对源语 文本中的错误, 翻译难度会更低, 同时, 译员自身也 会更从容自信, 在传统的无稿同传翻译活动中, 译员 要打起精神捕捉尽可能多的信息, 尤其是在不熟悉的 专业领域, 例如医药、化学、技术等主题的会议上, 在进入翻译活动之前译员就会开始担心自己当场的 发挥是否会打折扣, 遇到生疏的术语是否可以及时反 应过来。有了语音识别软件的辅助 “壮胆子”, 译员会 用更平常的心态对待专业程度较高的翻译活动, 焦虑 的减少有利于译员集中精神，平稳发挥。 


\section{2. 语音识别辅助对同声传译翻译过程的消 极影响}

\subsection{1. 分散注意力}

吉尔 (1995)、鲍刚 (2005)、卢信朝 (2009) 等 中外学者都对听辨理解进行了研究, 并指出了听辨在 口译中的重要性, 听辨是口译中首个关键环节, 在把 握不好主要和次要辅助工具, 不熟悉设备使用, 精力 分配不当时, 很容易出现注意力分散, 不清楚视线应 当集中在哪一辅助工具上, 导致听、看、译各个环节 都出现问题, 正如笔者在上述实验中出现的状况, 抓 不住注意力重点, 过于担心自己无法捕捉关键信息, 在听辨的同时还要核对屏幕内容, 会出现不当停顿等 问题, 影响整体的流畅度和句法结构。(Kenny，1996)

\subsection{2. 传递错误信息}

当译员过度依赖语音识别辅助工具, 对听辨放松 警惕, 会导致忽视源语转化中的错误, 传递错误信息。 例如由于发言人口音的缘故, 语义识别系统难以精确 识别源语, 容易呈现错误信息, 除此之外, 数字是翻 译中的一个难点, 需要翻译精确, 盲目相信语音识别 系统的转换容易造成大错。

\subsection{3. 造成译文几余}

同声传译过程中, 由于各门语言特点不同, 经常 出现翻译时间不够这样的问题。例如汉语到俄语的翻 译中, 由于俄语相对语句较长, 译员需要通过精简信 息、提出要点、提高译语语速等方法缩短翻译时间。 在单纯依靠笔记为主要辅助工具时, 译员会选取重要 信息进行传递, 达到简约流畅的效果。而语音识别系 统转换的文本过长, 阅读、识别、翻译所需时间也会 变长, 难以把握中心。并且, 同样时间内, 译文文本 变长就需要提高语速, 会给听众理解造成困难, 带来 不适感，使交流质量大打折扣。

\section{5. 结论}

翻译生态学理论（许建中, 2009) 认为, 译者和 翻译作品乃至参与翻译活动的各个主体, 都处在生态 选择和适应的过程之中, 适者生存法则同样适用于翻 译界。因此, 译员要有随着技术发展而发展的准备, 也要具备应对技术带来的挑战的能力。

人工智能的发展对翻译领域带来双重影响, 尤其 是在使用语音辅助技术时, 对翻译过程产生的影响取 决于具体状况。如合理分配精力, 恰当使用语音识别 的辅助作用, 可以减轻翻译过程的压力, 提高译文质 量, 保证译文完整性。如无法正确分配精力, 过度依 赖语音识别技术转发的源语文本, 则会出现分散注意 力、译文几余、传递错误信息等问题。因此, 语音识 别技术不断成熟的同时, 译员应慎重及恰当使用其辅
助功能，在该项技术的积极作用下提升翻译质量。

\section{REFERENCES}

[1] GILE D. (1995) Basic Concepts and Models in Interpreter and Translator Training. John Benjamins Publishing Company, Amsterdam/Philadelphia.

[2] Kenny K. (1996) Language Loss and the Crisis of Cognition. Mouton de Gruyter, Berlin.

[3] BAO GANG. (2010) Interpretation Studies. China Translation and Publishing Corporation, Beijing.

[4] LU XINCHAO. (2009) English Interpretation listening: Cognitive Psychological Model, Skills and Teaching, Shandong Foreign Language Teaching, 05 .

[5] LI TIANYUN. (2018) Analysis of Machine Simultaneous Interpretation System under Interpretation Working Model. Oriental Translation, 6: 34-39.

[6] WANG HAIKUN, PAN JIA, LIU CONG. (2018) Research Progress and Prospect of Speech Recognition Technology. Telecom Science, 2: 1-11.

[7] YU XIAOMING. (2019) Development and Application of Speech Recognition Technology. Computer Age, 11: 28-31.

[8] XU JIANZHONG. (2009) Translational Ecology. China Three Gorges Press, Beijing. 